\title{
Association between cardiovascular events and sodium-containing effervescent, dispersible, and soluble drugs: nested case-control study
}

\author{
() $(1) \Theta$ OPEN ACCESS
}

\author{
Jacob George senior lecturer/honorary consultant in clinical pharmacology ${ }^{1}$, Waseem Majeed core \\ medical trainee in medicine ${ }^{2}$, Isla S Mackenzie senior lecturer/honorary consultant in clinical \\ pharmacology ${ }^{3}$, Thomas M MacDonald professor of clinical pharmacology ${ }^{3}$, Li Wei senior lecturer \\ in medical statistics ${ }^{34}$
}

${ }^{1}$ Division of Medical Science, Ninewells Hospital and Medical School, Dundee DD1 9SY, UK; ${ }^{2} \mathrm{NHS}$ Tayside, Ninewells Hospital, Dundee DD1 9SY, UK; ${ }^{3}$ Medicines Monitoring Unit and Hypertension Research Centre, Ninewells Hospital and Medical School, Dundee DD1 9SY, UK; ${ }^{4}$ Department of Practice and Policy, UCL School of Pharmacy, London WC1H 9JP, UK

\begin{abstract}
Objective To determine whether patients taking formulations of drugs that contain sodium have a higher incidence of cardiovascular events compared with patients on non-sodium formulations of the same drugs. Design Nested case-control study.

Setting UK Primary Care Patients registered on the Clinical Practice Research Datalink (CPRD).

Participants All patients aged 18 or over who were prescribed at least two prescriptions of sodium-containing formulations or matched standard formulations of the same drug between January 1987 and December 2010.

Main outcome measures Composite primary outcome of incident non-fatal myocardial infarction, incident non-fatal stroke, or vascular death. We performed 1:1 incidence density sampling matched controls using the UK Clinical Practice Research Datalink (CPRD). For the secondary analyses, cases were patients with the individual components of the primary study composite endpoint of hypertension, incident heart failure, and all cause mortality.

Results 1292337 patients were included in the study cohort. Mean follow-up time was 7.23 years. A total of 61072 patients with an incident cardiovascular event were matched with controls. For the primary endpoint of incident non-fatal myocardial infarction, incident non-fatal stroke, or vascular death the adjusted odds ratio for exposure to sodium-containing drugs was 1.16 (95\% confidence interval 1.12 to 1.21). The adjusted odds ratios for the secondary endpoints were 1.22 (1.16 to 1.29$)$ for incident non-fatal stroke, 1.28 (1.23 to 1.33$)$ for all
\end{abstract}

cause mortality, 7.18 (6.74 to 7.65$)$ for hypertension, 0.98 (0.93 to 1.04) for heart failure, 0.94 (0.88 to 1.00$)$ for incident non-fatal myocardial infarction, and 0.70 (0.31 to 1.59) for vascular death. The median time from date of first prescription (that is, date of entry into cohort) to first event was 3.92 years.

Conclusions Exposure to sodium-containing formulations of effervescent, dispersible, and soluble medicines was associated with significantly increased odds of adverse cardiovascular events compared with standard formulations of those same drugs. Sodium-containing formulations should be prescribed with caution only if the perceived benefits outweigh these risks.

\section{Introduction}

Excess dietary sodium is a major public health problem worldwide. In response to a 2010 report by the Institute of Medicine, ${ }^{1}$ the National Salt Reduction Initiative (NSRI) was established with a goal to reduce dietary sodium consumption in the United States by $20 \%$ in five years. Reducing sodium intake in the US to the recommended $2.3 \mathrm{~g} /$ day $(100 \mathrm{mmol} / \mathrm{L}$ or one teaspoonful) could prevent 11 million cases of hypertension, save $\$ 18 \mathrm{bn}$ ( $£ 11 \mathrm{bn}, € 13 \mathrm{bn}$ ) healthcare dollars, and gain 312000 quality adjusted life years (QALYs) valued at $\$ 32 \mathrm{bn}$ annually. ${ }^{2}$ In the United Kingdom, the Food Standards Agency also launched a campaign in $2002^{3}$ to reduce salt intake in the estimated 26 million people in the UK who have high dietary sodium intake. It has been estimated that a $3 \mathrm{~g} / \mathrm{day}$ reduction in salt ( $1.2 \mathrm{~g} /$ day reduction in sodium) could prevent 
30000 cardiovascular events and save the National Health Service (NHS) at least $£ 40 \mathrm{~m} /$ year (€48m, $\$ 64 \mathrm{~m}){ }^{4}$

Excess sodium in healthy individuals impairs endothelial function, left ventricular relaxation, and cardiac repolarisation. ${ }^{5}$ Some of these effects could be independent of blood pressure in patients with hypertension. ${ }^{6}$ While considerable efforts are being made to reduce salt intake in the population in general we were concerned that a high sodium load can be ingested through certain formulations of medicines such as effervescent, dispersible, and soluble formulations. As an example, the recommended sodium intake for an adult in the UK is $2.4 \mathrm{~g} /$ day ( $104 \mathrm{mmol} / \mathrm{day})$. The dispersible and effervescent formulations of paracetamol $500 \mathrm{mg}$, however, can contain $18.6 \mathrm{mmol}$ and $16.9 \mathrm{mmol}$ of sodium in each tablet, respectively, and therefore the maximum daily dose of eight tablets/day results in the ingestion of $148.8 \mathrm{mmol}$ and $135.2 \mathrm{mmol}$ of sodium, respectively. This exceeds the recommended total daily allowance of sodium for one drug alone. Added to a typical Western diet, these drugs could result in high sodium intake. Curiously, unlike foods, pharmaceutical manufacturers are not placed under any restrictions or obligations with regards to sodium content or labelling of these sodium-containing formulations.

We hypothesised that sodium-containing formulations might increase adverse cardiovascular outcomes. We compared the risk of cardiovascular events in patients prescribed sodium-containing formulations with the risk in those prescribed standard tablet or capsular forms of the same drugs using the Antithrombotic Trialists' Collaboration (ATC) serious vascular event composite endpoint of non-fatal myocardial infarction, non-fatal stroke, or vascular death. ${ }^{7}$

\section{Methods}

\section{Study design}

This was a population based nested case-control study that used the UK Clinical Practice Research Datalink (CPRD) database. This is a large computerised database of anonymised longitudinal medical records derived from primary care. It contains data from over 500 primary care practices with 4.4 million active patients throughout the UK. The data have been collected since 1987 and cover about $7 \%$ of the UK population. The data from CPRD are validated and have been found to be generalisable to the whole UK population. ${ }^{8}$

\section{Study population}

The study population consisted of residents in the UK who were registered with their general practitioner and had more than one recorded contact from January 1987 to December 2010. Only general practices that met standards predefined by CPRD, registered as "up to standard" practices, were included in the study.

\section{Study patients}

The study population comprised all patients aged 18 or over who received at least two prescriptions of sodium-containing formulations or matched standard formulations of the same drug from January 1987 to December 2010. Within the category of sodium-containing formulations, we selected only drugs with more than 1000 prescriptions in the entire CPRD database.

\section{Study cohort}

Patients entered the study at the date of their first prescription for sodium or standard drug during the study period and were followed up until the end of December 2010. Patients were censored if they experienced an outcome event, died, or left their general practice during the study period or switched drugs. Patients were excluded from the study if they had a malignancy diagnosed (except basal cell carcinoma), malabsorption syndromes, salt wasting conditions, or a recorded history of alcohol/drug/substance abuse before the entry date. The above procedure was repeated for each individual outcome.

\section{Definition of cases and controls}

All patients with incident cardiovascular events during follow-up were identified by Read codes from the study cohort. Cardiovascular events were defined by the first occurrence of the Antithrombotic Trialists' Collaboration serious vascular event endpoint of non-fatal myocardial infarction, non-fatal stroke, or vascular death. ${ }^{7}$ For each case, we matched one control with incidence density sampling on year of birth, sex, and general practice attended. The date of the first cardiovascular event during the follow-up was defined as the index date for each case and was used for the index date for the matched controls. The above case and control selection procedure was repeated for the individual components of the ATC event endpoint: hypertension, incident heart failure, and all cause mortality.

\section{Drug treatments}

We identified 24 different drugs with sodium-containing formulations (dispersible, effervescent, and soluble) and a further 116 different comparable standard formulations that were non-dispersible, non-effervescent, or non-soluble versions of the 24 sodium-containing drugs. Table 1 shows examples of sodium-containing formulations $\Downarrow$. The sodium content for each sodium-containing formulation was obtained from the summary of product characteristics or by contacting the individual manufacturers when this information was not found in the summary.

\section{Covariates}

The covariates included age, sex, body mass index (BMI), smoking status, hypertension, peripheral vascular disease, angina, chronic obstructive pulmonary disease (COPD), diabetes mellitus, chronic kidney disease, migraine, heart failure, oesophageal disease (which might make the prescription of soluble/effervescent drugs more likely), and prescriptions of potassium sparing diuretics (because of potassium's potential beneficial vasoactive effects ${ }^{9}$ ).For co-prescribed drugs, covariates adjusted for in the model included angiotensin converting enzyme inhibitors or angiotensin receptor blockers, proton pump inhibitors, oral steroids, non-steroidal anti-inflammatory drugs (NSAIDs) or COX-2 inhibitors, antipsychotic drugs, anti-HIV therapy, statins, and antiplatelets.

\section{Statistical analysis}

Data are presented as mean (SD) for continuous variables and as numbers (\%) for categorical variables. We performed $\chi^{2}$ tests and analysis of variance to determine significant differences. Conditional logistic regression analysis was used to determine odds ratios for the association between patients prescribed sodium-containing formulations and the risk of cardiovascular outcomes as well as all cause mortality. We also carried out univariate and multivariate analyses. In the multivariate models we adjusted the odd ratios for all covariates between the study groups. Appendix 1 shows a stepwise analysis for the primary outcome and a correlation matrix for the factors analyses. Data 
were missing for $14 \%$ for smoking and $28 \%$ for BMI. The multiple imputation analysis included all variables used in the final analysis with 10 imputations by using the Markov chain Monte Carlo method to produce imputed data. We carried out sensitivity analyses to deal with the missing data mechanisms. A complete case analysis was conducted (see appendix 2 for details). All results were expressed as odds ratios (95\% confidence intervals). All statistical analyses were carried out with SAS (version 9.2).

\section{Results}

\section{Patients' characteristics}

We included 1292337 patients in the study. The mean follow-up time was 7.23 years. During follow-up 61072 events that met the definition of the primary cardiovascular endpoint occurred. The median time from date of first prescription (that is, date of entry into cohort) to first occurrence of non-fatal myocardial infarction, non-fatal stroke, or vascular death was 3.92 years. Table $2 \Downarrow$ shows the baseline characteristics between the groups. The cohort of cases had significantly more (all $\mathrm{P}<0.01)$ smokers, history of angina, heart failure, chronic obstructive pulmonary disease, peripheral vascular disease, diabetes mellitus, and chronic kidney disease. Likewise there were also more prescriptions for drugs for cardiovascular disease, NSAIDs, and potassium supplements than in the control group. All these parameters, however, were included in and adjusted for in the final analysis.

\section{Primary endpoint}

The 61072 cases were matched with the same number of controls in the primary analysis. Compared with standard formations, the odds ratio adjusted for demographic factors (smoking and BMI) for sodium rich drug formulations was 1.14 (95\% confidence interval 1.09 to 1.18 ). The fully adjusted exposure odds ratio for all covariates for sodium-rich drug formulations for the cases was 1.16 (1.12 to 1.21) compared with controls.

\section{Secondary endpoint}

When we examined individual components of the composite endpoint (table $3 \Downarrow$ ), adjusted exposure odds ratios in cases were 0.94 (0.88 to 1.00$)$ for incident non-fatal myocardial infarction, 1.22 (1.16 to 1.29) for incident non-fatal stroke, and 0.70 (0.31 to 1.59$)$ for vascular death. For other secondary endpoints adjusted odds ratios were 7.18 (6.74 to 7.65$)$ for hypertension, 0.98 ( 0.93 to 1.04 ) for incident heart failure, and 1.28 (1.23 to 1.33) for all cause mortality.

\section{Effect of cumulative dose}

Compared with controls the adjusted odds ratios were 1.10 (0.99 to 1.22 ) for the lowest third ( $\leq 7120$ mmol sodium), 1.33 (1.20 to 1.48 ) for the middle third (7121-30 $285 \mathrm{mmol}$ sodium), and 1.31 (1.18 to 1.45 ) for the high third (>30 285 mmol sodium). A test for linear trend showed there was significant trend in the dose-response relation $(\mathrm{P}<0.01)$.

\section{Further analysis}

To explore these findings further, we also performed a cohort study. The sodium cohort was defined as people who had at least two prescriptions for sodium-containing effervescent, dispersible or soluble formulations during the study period (table $1 \Downarrow)$ and the standard cohort was defined as those who had at least two prescriptions for non-dispersible, non-effervescent, or non-soluble versions of the drugs used by the sodium cohort during the study period.

Compared with the standard cohort, the unadjusted and adjusted hazard ratios for the composite endpoint in the sodium cohort were 1.24 (95\% confidence interval 1.20 to 1.29$)$ and $1.17(1.13$ to 1.21), which supports the results of the case-control analysis. Further analysis in which we used only those patients without any missing data $(n=68224)$ showed an adjusted hazard ratio of 1.20 (1.12 to 1.27 ), which was similar to the main result.

\section{Discussion}

\section{Principal findings}

Patients who experienced the composite outcome of primary outcome of incident non-fatal myocardial infarction, incident non-fatal stroke, or vascular death were more likely to have been prescribed sodium-containing formulations of drugs compared with people who had not experienced this outcome (controls). Subsequent secondary analyses showed that the increased risk of stroke was the component of the composite endpoint that was driving the primary outcome (odds ratio 1.20, 95\% confidence interval 1.15 to 1.26 ). This increased risk of stroke was probably associated with the increased risk of hypertension (7.18, 6.74 to 7.65$)$. The exposure odds ratio for all cause mortality was also significantly higher $(1.29,125$ to 1.34).

The significantly increased exposure odds ratio for stroke is consistent with the results of a recently published meta-analysis of sodium exposure.$^{10}$ Furthermore, it has been previously shown that dietary sodium contributes significantly to resistance to antihypertensive treatment. ${ }^{11}$ The increased exposure odds ratio for incident stroke risk is consistent with numerous published studies of sodium intake including a recent meta-analysis. ${ }^{12}$ Likewise, the increase in exposure odds ratio for all cause mortality is consistent with the findings of the Third National Health and Nutrition Examination Survey (NHANES III), in which higher sodium intake was associated with increased all cause mortality (hazard ratio 1.20,95\% confidence interval 1.03 to 1.41 , per $1000 \mathrm{mg} /$ day. ${ }^{13}$ Thus, we have shown significant and biologically plausible serious adverse effects of sodium-containing prescribed drugs compared with standard drugs.

To put these findings into context, the median sodium consumption from sodium-containing drugs alone in our study was $106.8 \mathrm{mmol} /$ day. This amount is higher than the current recommended dietary intake of $104 \mathrm{mmol} /$ day.

We did not observe an increase in the incidence of heart failure, despite an increase in hypertension. A recent review of the currently available evidence, however, suggests that the association between sodium intake and incident heart failure is not as clear cut as one might assume. ${ }^{14}$ Indeed, several studies have found a worsening outcome with sodium restriction because of neurohumoral activation.

\section{Comparison with other studies}

We acknowledge that there is still some controversy regarding the relation between dietary sodium and cardiovascular events. Some meta-analyses and systematic reviews on the effect of dietary sodium on cardiovascular health have reached opposite conclusions. ${ }^{10}{ }^{15}$ Even large surveys such as NHANES $\mathrm{I}^{16}$ and $\mathrm{II}^{17}$ have shown conflicting results with regards to the relation between dietary sodium and cardiovascular events. Some researchers have since suggested that there might either be an inverse or a $\mathbf{J}$ shaped curve relation between dietary sodium and 
event rates for cardiovascular disease. ${ }^{18}$ Although we found an increased risk of incident hypertension, incident stroke, and all cause mortality in patients prescribed sodium-containing drugs, we did not detect a significant increase in the risk of myocardial infarction or vascular death. This study therefore supports the effect of sodium-induced hypertension, through ingested drugs, on the incidence of stroke.

It might be that taking sodium-containing formulations is associated with less good health behaviour and that this led to fewer contacts with health professionals. This seems unlikely, however, as initiating a prescription for a sodium-containing drug itself would necessitate contact with a general practitioner. Our data were adjusted for recorded smoking, alcohol intake, and deprivation scores, but we could not determine other aspects of health behaviour such as adherence to treatment or poor diet. This requires further exploration. We adjusted for other covariates, such as oesophageal disease, as described in the methods section.

We believe that our findings are potentially of public health importance. The sodium content of medicines seems to be an important topic that needs to be dealt with by regulatory agencies. As a minimum, the public should be warned about the potential hazards of high sodium consumption in prescribed medicines, and these should be clearly labelled with the sodium content in the same way as foods are labelled. Although we did not study non-prescription or over-the-counter medicines, we think that it is reasonable to extrapolate our findings to these medicines. Arguably, over-the-counter preparations that have more questionable therapeutic benefit should have their risk-benefit balance reassessed. Pharmaceutical innovation to reduce the sodium content of drug formulations while preserving the desired effervescent, soluble, or dispersible characteristics would be a wholly desirable goal.

The relation between salt intake and cardiovascular risk in humans was recognised 60 years ago when Lewis Dahl proposed that blood pressure rises linearly with salt intake. ${ }^{19}$ The strength of this relation has been shown in large studies such as the International Study of Salt and Blood pressure (INTERSALT study), involving more than 10000 patients; in populations with a high salt intake the relation between blood pressure and age was stronger than in populations with a low salt intake. ${ }^{20}$

Despite the overwhelming evidence of the effect of dietary sodium on blood pressure and cardiovascular risk, there is currently little clinical evidence on the effect of widely used sodium-containing formulations such as effervescent, dispersible, and soluble drugs on patient health. One small pilot study looked at the effect of sodium-containing formulations on blood pressure in elderly patients with hypertension and uncontrolled blood pressure treated with effervescent paracetamol ( $3 \mathrm{~g} /$ day) for osteoarthritis. ${ }^{21}$ Blood pressure was measured before and after (four weeks or more) switching paracetamol from the effervescent formulation to a standard formulation. Switching to standard paracetamol tablets was associated with $13.1 / 2.5 \mathrm{~mm} \mathrm{Hg}$ reduction in blood pressure. This study provides another biologically plausible mechanism to support a causal association between prescribed sodium-containing formulations and increased cardiovascular risk.

\section{Strengths}

This was a large general practice population database study with a mean follow-up of 7.23 years. As such it is likely to reflect usual healthcare in the UK. We studied cardiovascular events rather than an intermediate surrogate measure. Compared with a time dependent survival analysis, a nested case-control analysis has superior computational efficiency ${ }^{22}$ while producing odds ratios that are unbiased estimators of incidence rate ratios, with little or no loss in precision ${ }^{23}$

\section{Limitations}

There might be coding misclassification for the exposures, outcomes, and covariates in the database. The present study is observational and other potential confounding factors and biases could not be fully controlled. Data on unmeasured or unmeasurable risk factors such as health behaviour and family history were not available. We had no data on dietary sodium and could not control for this. Finally, we could not control for medicines bought over-the counter. On the basis that people who take sodium-containing formulations do so for a reason it is likely that consumption of over-the-counter drugs would mirror prescribed formulations and this could tend to amplify the total sodium load.

\section{Conclusions and policy implications}

Patients experiencing incident non-fatal myocardial infarction, incident non-fatal stroke, or vascular death (cases) had a greater exposure to sodium-containing effervescent, dispersible, and soluble drugs than controls. The primary outcome was largely driven by stroke, which is consistent with the increased risk of hypertension shown. The odds ratio for all cause mortality was also increased with these sodium-containing drugs. Our results suggest that physicians should prescribe sodium-containing formulations with caution and only if there are compelling reasons to do so. Sodium loaded effervescent, soluble, or dispersible tablets should be avoided in patients at risk of hypertension, and patients prescribed these drugs should be carefully monitored for the emergence of hypertension.

Contributors: JG conceived the idea for the study. JG, TMM, and LW obtained funding for the study. JG, TMM, ISM, and LW were involved in design, analysis, and interpretation of data. JG, WM, and IS were involved in data collection. All authors were responsible for drafting the article or revising it critically for important intellectual content and approved the final version. LW is guarantor.

Funding: This study was funded by TENOVUS Scotland (ref T10/12). The funder had no role in the design, conduct or data interpretation of the study.

Competing interests: All authors have completed the ICMJE uniform disclosure form at www.icmje.org/coi_disclosure.pdf and declare: no support from any organisation for the submitted work; no financial relationships with any organisations that might have an interest in the submitted work in the previous three years; no other relationships or activities that could appear to have influenced the submitted work.

Ethical approval: This study was approved by the independent scientific advisory committee (ISAC) for the UK Medicines and Healthcare Products Regulatory Agency (MHRA) database research (protocol No 11_100).

Transparency: The lead author affirms that the manuscript is an honest, accurate, and transparent account of the study being reported; that no important aspects of the study have been omitted; and that any discrepancies from the study as planned (and, if relevant, registered) have been explained.

Data sharing: No additional data available.

Institute of Medicine. Strategies to reduce sodium intake in the United States. Institute of Medicine, 2010.

2 Palar K, Sturm R. Potential societal savings from reduced sodium consumption in the U.S. adult population. Am J Health Promot 2009;24:49-57. 


\section{What is already known on this topic}

Numerous observational studies have shown that excess salt is detrimental to cardiovascular health

The effect of commonly prescribed sodium-containing effervescent, dispersible, and soluble drugs on cardiovascular events is unknown

\section{What this study adds}

Patients prescribed sodium-containing effervescent, dispersible and soluble formulations of medications have an excess of cardiovascular events compared with patients prescribed identical drugs without the sodium

These events are largely driven by an increased risk of hypertension and stroke

Prescription of these sodium-containing formulations should be done with caution, and patients prescribed them should be closely monitored for the emergence of hypertension

3 Food Standards Agency. Health initiative worth its salt, says Agency. Food Standards Agency, 2002. http://tna.europarchive.org/20110116113217/http://www.food.gov.uk/news/ newsarchive/2002/jan/salt_awareness_day

4 Barton P, Andronis L, Briggs A, McPherson K, Capewell S. Effectiveness and cost effectiveness of cardiovascular disease prevention in whole populations: modelling study. BMJ 2011;343:d4044.

5 Tzemos N, Lim PO, Wong S, Struthers AD, MacDonald TM. Adverse cardiovascular effects of acute salt loading in young normotensive individuals. Hypertension 2008;51:1525-30.

6 Lim PO, Farquharson CA, Shiels P, Jung RT, Struthers AD, MacDonald TM. Adverse cardiac effects of salt with fludrocortisone in hypertension. Hypertension 2001;37:856-61.

7 Antithrombotic Trialists' Collaboration. Collaborative meta-analysis of randomised trials of antiplatelet therapy for prevention of death, myocardial infarction, and stroke in high risk patients. BMJ 2002;324:71-86.

8 Herrett E, Thomas SL, Schoonen WM, Smeeth L, Hall AJ. Validation and validity of diagnoses in the General Practice Research Database: a systematic review. Br J Clin Pharmacol 2010;69:4-14.

9 Haddy FJ, Vanhoutte PM, Feletou M. Role of potassium in regulating blood flow and blood pressure. Am J Physiol Regul Integr Comp Physiol 2006;290:R546-52.

10 Aburto NJ, Ziolkovska A, Hooper L, Elliott P, Cappuccio FP, Meerpohl JJ. Effect of lower sodium intake on health: systematic review and meta-analyses. BMJ 2013;346:f1326.

11 Pimenta E, Gaddam KK, Oparil S, Aban I, Husain S, Dell'Italia LJ, et al. Effects of dietary sodium reduction on blood pressure in subjects with resistant hypertension: results from a randomized trial. Hypertension 2009;54:475-81.

12 Li XY, Cai XL, Bian PD, Hu LR. High salt intake and stroke: meta-analysis of the epidemiologic evidence. CNS Neurosci Ther 2012;18:691-701.

13 Yang Q, Liu T, Kuklina EV, Flanders WD, Hong Y, Gillespie C, et al. Sodium and potassium intake and mortality among US adults: prospective data from the Third National Health and Nutrition Examination Survey. Arch Intern Med 2011;171:1183-91.

14 Gupta D, Georgiopoulou VV, Kalogeropoulos AP, Dunbar SB, Reilly CM, Sands JM, et al. Dietary sodium intake in heart failure. Circulation 2012;126:479-85.
15 Graudal NA, Galloe AM, Garred P. Effects of sodium restriction on blood pressure, renin, aldosterone, catecholamines, cholesterols, and triglyceride: a meta-analysis. JAMA 1998:279:1383-91.

16 Alderman $\mathrm{MH}$, Cohen $\mathrm{H}$, Madhavan S. Dietary sodium intake and mortality: the National Health and Nutrition Examination Survey (NHANES I). Lancet 1998;351:781-5.

17 Cohen HW, Hailpern SM, Fang J, Alderman MH. Sodium intake and mortality in the NHANES II follow-up study. Am J Med 2006;119:275.e7-14.

18 Alderman $\mathrm{MH}$, Cohen HW. Dietary sodium intake and cardiovascular mortality: controversy resolved? Curr Hypertens Rep 2012;14:193-201.

19 Dahl LK, Love RA. Evidence for relationship between sodium (chloride) intake and human essential hypertension. AMA Arch Intern Med 1954;94:525-31.

20 Intersalt Cooperative Research Group. Intersalt: an international study of electrolyte excretion and blood pressure. Results for 24 hour urinary sodium and potassium excretion. BMJ 1988:297:319-28.

21 Ubeda A, Llopico J, Sanchez MT. Blood pressure reduction in hypertensive patients after withdrawal of effervescent medication. Pharmacoepidemiol Drug Saf 2009;18:417-9.

22 Essebag V, Platt RW, Abrahamowicz M, Pilote L. Comparison of nested case-control and survival analysis methodologies for analysis of time-dependent exposure. BMC Med Res Methodol 2005;5:5.

23 Azoulay L, Dell'aniello S, Simon T, Renoux C, Suissa S. The concurrent use of antithrombotic therapies and the risk of bleeding in patients with atrial fibrillation. Thromb Haemost 2013;109:431-9.

Accepted: 13 November 2013

\section{Cite this as: BMJ 2013;347:f6954}

This is an Open Access article distributed in accordance with the Creative Commons Attribution Non Commercial (CC BY-NC 3.0) license, which permits others to distribute, remix, adapt, build upon this work non-commercially, and license their derivative works on different terms, provided the original work is properly cited and the use is non-commercial. See: http://creativecommons.org/licenses/by-nc/3.0/. 


\section{Tables}

\section{Table 1| Examples of sodium content per tablet and maximum daily ingested sodium for selected sodium-containing formulations}

\section{Formulation}

Paracetamol soluble tablet $500 \mathrm{mg}$

Paracetamol with codeine phosphate effervescent tablet $500 \mathrm{mg}+30 \mathrm{mg}$

Co-codamol sugar-free effervescent powder $30 \mathrm{mg}+500 \mathrm{mg}$

Paracetamol with codeine phosphate effervescent tablet $500 \mathrm{mg}+8 \mathrm{mg}$

Paracetamol with codeine phosphate effervescent tablet $500 \mathrm{mg}+30 \mathrm{mg}$ (preparation 1)

Paracetamol with codeine phosphate effervescent tablet $500 \mathrm{mg}+30 \mathrm{mg}$ (preparation 2)

Metoclopramide with aspirin effervescent tablet $5 \mathrm{mg}+325 \mathrm{mg}$

Metoclopramide with aspirin effervescent tablet $5 \mathrm{mg}+450 \mathrm{mg}$

Ibuprofen soluble tablet $200 \mathrm{mg}$

Ascorbic acid effervescent tablet $1 \mathrm{~g}$

Aspirin effervescent tablet $300 \mathrm{mg}$

Calcium lactate gluconate with calcium carbonate effervescent tablets $2263 \mathrm{mg}+1750 \mathrm{mg}$

Zinc sulphate sugar-free effervescent tablets $125 \mathrm{mg}$

Zinc sulphate monohydrate effervescent tablet $125 \mathrm{mg}$

Calcium gluconate effervescent tablet $1 \mathrm{~g}$

Paracetamol soluble tablet $120 \mathrm{mg}$

Calcium carbonate with colecalciferol effervescent tablet $1500 \mathrm{mg}+10 \mu \mathrm{g}$

Calcium carbonate effervescent tablet $1.25 \mathrm{~g}$

Calcium carbonate and colecalciferol effervescent granules $1250 \mathrm{mg}+440 \mathrm{IU}$

Calcium carbonate + colecalciferol effervescent granules $1.25 \mathrm{~g}+11 \mu \mathrm{g}$

$\mathrm{Na}^{+}$per tablet (mmol) Maximum daily $\mathrm{Na}^{+}(\mathrm{mmol} / \mathrm{day})$ 148.8

$17.8 \quad 142$.

$17.8 \quad 142.4$

$16.9 \quad 135.2$

16.9

14.2113 .6

$12.0 \quad 72.0$

$12.0 \quad 48.0$

$8.8 \quad 52.8$

$8.4 \quad 8.4$

$6.5 \quad 6.5$

$6.0 \quad 6.0$

$4.6 \quad 13.8$

$\begin{array}{ll}4.6 & 13.8\end{array}$

$4.5 \quad 36.0$

$3.5 \quad 56.0$

$2.3 \quad 4.6$

$0.9 \quad 1.8$

$0.2 \quad 0.4$

$\begin{array}{ll}0.2 & 0.4 \\ 0.2 & 0.4\end{array}$


Table 2| Baseline characteristics of patients in UK Clinical Practice Research Datalink database with individual components of primary study composite endpoint of hypertension, incident heart failure, and all cause mortality (cases) and matched controls. Figures are numbers (percentage) of patients unless stated otherwise

\begin{tabular}{|c|c|c|c|}
\hline & Cases $(n=61072)$ & Controls $(n=61072)$ & $P$ value \\
\hline Mean (SD) age (years) & $69(12.3)$ & $69(12.4)$ & - \\
\hline Men & $25281(41)$ & $25281(41)$ & - \\
\hline \multicolumn{4}{|l|}{ BMI: } \\
\hline$<18.5$ & $886(2)$ & $782(1)$ & \multirow[t]{4}{*}{$<0.01$} \\
\hline $18.5-24.9$ & $15287(25)$ & $15354(25)$ & \\
\hline $25-29.9$ & $17618(29)$ & $17536(29)$ & \\
\hline$\geq 30$ & $10611(17)$ & $9904(16)$ & \\
\hline Missing & $16670(27)$ & $17496(22)$ & - \\
\hline \multicolumn{4}{|l|}{ Smoking status: } \\
\hline None & $8120(13)$ & $9732(16)$ & \multirow[t]{3}{*}{$<0.01$} \\
\hline Current smoker & $11288(19)$ & $8597(14)$ & \\
\hline Ex-smoker & $26685(44)$ & $29068(48)$ & \\
\hline Missing & $14979(25)$ & $16375(22)$ & - \\
\hline \multicolumn{4}{|l|}{ Disease history: } \\
\hline Angina & $11653(19)$ & $7373(12)$ & $<0.01$ \\
\hline Heart failure & $4606(8)$ & $3373(6)$ & $<0.01$ \\
\hline Peripheral vascular disease & $2507(4)$ & $1470(2)$ & $<0.01$ \\
\hline Migraine & $3188(5)$ & $2764(5)$ & $<0.01$ \\
\hline Oesophageal disease & $136(0.22)$ & $108(0.2)$ & 0.06 \\
\hline Diabetes & $9485(16)$ & $6848(11)$ & $<0.01$ \\
\hline Chronic kidney disease & $5234(89)$ & $4672(7)$ & $<0.01$ \\
\hline COPD & $6113(10)$ & $5325(9)$ & $<0.01$ \\
\hline \multicolumn{4}{|l|}{ Drug history: } \\
\hline Cardiovascular disease drugs & $44957(74)$ & $35497(58)$ & $<0.01$ \\
\hline ACE/ARB & $21666(36)$ & $17183(28)$ & $<0.01$ \\
\hline Proton pump inhibitors & $22381(37)$ & $20562(34)$ & $<0.01$ \\
\hline Potassium & $2811(5)$ & $2154(4)$ & $<0.01$ \\
\hline Antipsychotic & $16784(28)$ & $14932(25)$ & $<0.01$ \\
\hline HIV & $3(0)$ & $1(0)$ & $<0.01$ \\
\hline Steroids & $4486(7)$ & $3924(6)$ & $<0.01$ \\
\hline NSAIDs & $40940(67)$ & $42299(69)$ & $<0.01$ \\
\hline
\end{tabular}

$\mathrm{BMI}=$ body mass index; $\mathrm{COPD}=$ chronic obstructive pulmonary disease; $\mathrm{ACE} / \mathrm{ARB}=$ angiotensin converting enzyme inhibitors/angiotensin receptor blockers; NSAIDs=non-steroidal anti-inflammatories. 
Table 3 | Odds ratios and $95 \%$ confidence intervals for composite cardiovascular outcome ${ }^{\star}$ and individual outcomes for sodium-containing formulations group compared with standard formulations group (OR=1)

\begin{tabular}{lll} 
& \multicolumn{2}{c}{ OR $(95 \% \mathrm{Cl})$} \\
\cline { 2 - 3 } & \multicolumn{1}{c}{ Unadjusted } & \multicolumn{1}{c}{ Adjusted $\boldsymbol{}$} \\
\hline Composite cardiovascular outcome* & $1.13(1.09$ to 1.18$)$ & $1.16(1.12$ to 1.21$)$ \\
\hline Individual outcomes & & \\
\hline Incident non-fatal myocardial infarction & $0.90(0.85$ to 0.96$)$ & $0.94(0.88$ to 1.00$)$ \\
\hline Incident non-fatal stroke & $1.21(1.15$ to 1.28$)$ & $1.22(1.16$ to 1.29$)$ \\
\hline Vascular death & $0.62(0.31$ to 1.24$)$ & $0.70(0.31$ to 1.59$)$ \\
\hline Hypertension & $6.80(6.41$ to 7.21$)$ & $7.18(6.74$ to 7.65$)$ \\
\hline Heart failure & $0.95(0.91$ to 1.00$)$ & $0.98(0.93$ to 1.04$)$ \\
\hline All cause mortality & $1.30(1.25$ to 1.35$)$ & $1.28(1.23$ to 1.33$)$ \\
\hline
\end{tabular}

*Incident non-fatal myocardial infarction, incident non-fatal stroke, and vascular death.

†See methods sections for details of adjustment. 